\title{
Rapid Evaluation after High-Risk TIA is Associated with Lower Stroke Risk
}

\author{
Caren M. Wu, Braden J. Manns, Michael D. Hill, William A. Ghali, \\ Cam Donaldson, Alastair M. Buchan
}

\begin{abstract}
Background: Current 'standard of care' for patients presenting with a 'high-risk' TIA varies, with use of several outpatient and inpatient approaches. We describe the clinical outcomes and costs for high risk TIA patients who received care in a 'rapid evaluation unit', and compare these to a historical 'high-risk' cohort. Methods: The study cohort was comprised of patients with TIA admitted to a 'rapid evaluation unit' during the period March 2002 to April 2003. The comparison cohort was established by screening Calgary Health Region ER discharge records to identify all patients presenting with a diagnosis of TIA during the year 2000. A 'highrisk standard care cohort' was then identified based on the clinical admission criteria used to select patients for the rapid evaluation unit. Outcomes (stroke within 90 days, death) and costs were identified using chart review and provincial administrative data. Results: The early risk of stroke in the high risk standard care group (392 patients) was 9.7\%, compared to $4.7 \%$ in the rapid evaluation cohort (189 patients) ( $\mathrm{p}=0.05)$. Median 1-year costs post TIA were CAN $\$ 8360$ for patients in the rapid evaluation cohort, compared with CAN $\$ 4820$ for patients in the high risk standard care group $(\mathrm{p}<0.001)$. Conclusions: The risk of early stroke was lower for patients in the rapid evaluation cohort compared to the high risk standard care cohort, suggesting that the use of rapid evaluation programs in patients with TIA at high risk of stroke may be beneficial, but incur greater costs over the course of the first year.
\end{abstract}

RÉSUMÉ: Une évaluation rapide après une ICT à haut risque est associée à un risque plus faible d'accident vasculaire cérébral. Résumé : Contexte : Actuellement, les «normes de soins établies » pour les patients qui consultent pour une ICT à haut risque varient et différentes lignes de conduite chez les patients externes et les patients hospitalisés sont préconisées. Nous décrivons les résultats cliniques et les coûts chez les patients atteints d'ICT à haut risque qui reçoivent des soins dans une « unité d'évaluation rapide» et nous comparons ces cas à une cohorte historique «à haut risque ». Méthodes : La cohorte de sujets était constituée de patients ayant consulté pour une ICT qui ont été évalués dans une « unité d'évaluation rapide » entre mars 2002 et avril 2003. La cohorte témoin a été constituée par révision des dossiers de patients qui ont reçu leur congé de l'unité des urgences de la Calgary Health Region pour identifier tous les patients chez qui un diagnostic d'ICT a été posé au cours de l'année 2000. Selon les critères cliniques d'admission utilisés pour choisir les patients pour l'unité d'évaluation rapide, une cohorte de patients à haut risque qui a reçu des soins standards a été identifiée. L'issue (accident vasculaire cérébral dans les 90 jours, décès) et les coûts ont été déterminés par une revue des dossiers et des données administratives provinciales. Résultats : Le risque précoce d'accident vasculaire cérébral dans le groupe à haut risque qui a reçu des soins standards (392 patients) était de $9,7 \%$ comparé à $4,7 \%$ dans la cohorte qui a bénéficié de l'évaluation rapide (189 patients) $(\mathrm{p}=0,05)$. Un an après l’ICT, les coûts étaient de 8360 $\$ C A$ pour les patients de la cohorte d'évaluation rapide et de 4821 \$CA pour les patients du groupe de soins standards de patients à haut risque (p < 0,001). Conclusions : Le risque d'un AVC précoce était plus faible chez les patients de la cohorte d'évaluation rapide par rapport à la cohorte de soins standards chez des patients à haut risque, ce qui suggère que l'utilisation de programmes d'évaluation rapide chez les patients présentant une ICT à haut risque d'AVC puisse être bénéfique mais génère des coûts plus élevés au cours de la première année.

Can. J. Neurol. Sci. 2009; 36: 450-455

Approximately $15 \%$ of ischemic strokes are preceded by transient ischaemic attack (TIA) ${ }^{1}$. Risk of stroke after TIA is higher than was previously thought, with more recent reports estimating risk of stroke within 90 days of TIA ranging between approximately $6-10 \% .^{2-4}$ We recently performed a systematic review and meta-analysis of studies reporting early risk of stroke after TIA and demonstrated a pooled risk of $9.2 \%$ at 90 days. ${ }^{5}$

There is growing consensus regarding the need for early stratification and management of patients presenting with a TIA. There have been no randomized clinical trials addressing the efficacy of rapid evaluation and treatment of TIA. Recent observational studies suggest that rapid evaluation and treatment of these patients in a 'TIA clinic' usually within 24 hours of their event may reduce recurrent stroke by up to $80 \% .^{6,7}$
The Calgary Stroke Program initiated a rapid admission and evaluation unit for patients with high-risk TIA in 2002. Patients within 24 hours of their TIA who have pre-defined characteristics suggesting they are high risk for early stroke are

From the Department of Medicine (CMW, BJM,WAG), Department of Community Health Sciences (BJM, WAG, CD), Department of Clinical Neurosciences (MDH,AMB), University of Calgary, Calgary, Alberta, Canada; Institute of Health and Society (CD), Newcastle University, Newcastle Upon Tyne; Medical Sciences Division (AMB), University of Oxford, Oxford, United Kingdom.

Received November 19, 2008. Final Revisions Submitted February 26, 2009. Correspondence to: Caren M. Wu, University of Calgary, Health Sciences Centre, 3330 Hospital Drive, Room 1443, Calgary Alberta, T2N 4N1, Canada. 
admitted to this rapid evaluation unit to receive diagnostic services and early triage to appropriate therapy.

Given that health care resources are scarce, it is important to know not only the effectiveness of a new program but also its costs. In this study, we describe the clinical outcomes and resource requirements associated with a rapid evaluation unit for high risk patients with TIA and compare it with outcomes and costs for patients who received prior 'standard care'.

\section{METHODS}

Ethical approval was obtained for the study from the Conjoint Research Ethics Board. Given that we wished to compare the clinical outcomes and costs for similar TIA patients receiving rapid evaluation and standard care, we sought to identify comparable cohorts using a population-based approach including all patients presenting with TIA within the Calgary Health Region over two separate time periods.

Patients in the rapid evaluation cohort were those who were referred (almost always from the emergency room) to the rapid evaluation and admission unit for TIA between the dates of March 9th 2002 and April 1, 2003. The inclusion criteria for patients admitted to the rapid evaluation and admission unit were as follows: TIA within 24 hours, a hemispheric event (hemiparesis, aphasia) or monocular blindness. All eligible patients were admitted. Explicitly, patients with purely sensory events, isolated dysarthria or vertigo were discharged from the Emergency Department to be investigated in the outpatient stroke clinic.

For the standard care cohort, the population of interest included all patients who presented to Emergency rooms in the Calgary Health Region in 2000 and were given the discharge diagnosis of TIA as identified using ICD-9 codes for TIA (435.x, "transient cerebral ischemia"). Further to this, patients from the standard care cohort were classified into a 'high-risk' subgroup, on the basis of meeting the admission criteria noted above for the rapid evaluation unit.

For patients in both cohorts, we confirmed the diagnosis of TIA using the World Health Organization definition of TIA as follows: rapidly developed clinical signs of focal or global disturbance of cerebral function lasting fewer than 24 hours, with no apparent nonvascular cause. Patients who presented with irreversible neurological deficits, who by definition did not have TIA and had completed their stroke, were excluded. In addition, patients who were from out-of province or out-of country were excluded as it was not possible to obtain the necessary follow-up clinical outcome or cost data.

Chart abstraction was performed for all patients in the study for both cohorts to collect data on patient demographics, TIA symptom complex and duration, including etiology of TIA if known, co-morbid conditions, baseline medications, and disposition from the ER. Data on management, including investigations and changes to medications were collected as well as use of interventions such as carotid angioplasty and endarterectomy.

The primary outcome of our study, stroke occurrence after TIA, was determined by surveillance of provincial administrative data and through review of hospital records. Codes used to identify stroke for patients in the standard care cohort included ICD-9 codes 433.x (occlusion and stenosis of cerebral arteries), 434.x (occlusion of cerebral arteries) and 436.x (acute but ill-defined cerebrovascular disease). For patients in the rapid evaluation cohort, due to the transition to use of ICD-10 codes in 2001, we used the matching ICD-10 codes (I63-I64) to identify potential strokes after TIA. For all early strokes that were noted using administrative data only, the diagnosis of stroke was subsequently confirmed through review of hospital records. The assignment was based on imaging findings as well as clinical description from the chart and was adjudicated by two physicians, a neurologist (MDH) and general internist (CMW). Of these, three instances were identified in the standard care cohort as not being true stroke occurrences and these were excluded. All others were validated.

\section{Statistical Analysis}

Fisher's exact tests were used to compare baseline characteristics between the study cohorts. Subsequently, the standard care cohort and rapid evaluation cohorts were combined for analysis using logistic regression controlling for exposure and other baseline characteristics to predict the association of type of care with early stroke. All variables of interest were initially tested in uni-variable models for significance. Subsequently, significant variables were included in the model with stepwise backwards elimination of variables using likelihood ratio tests. Several interaction terms were considered but none were significant.

For the cost analysis, the perspective was that of the health care payer. We obtained direct health care costs from Alberta Health and Wellness, categorized into inpatient and outpatient care, physician payments, and outpatient medications for one year after TIA. Costs for homecare and nursing home admissions, as well as indirect costs (i.e. patient time costs, etc) were not included. Costs were reported in 2002 CAN \$. STATA 7.0 was used for all analyses.

\section{RESULTS}

\section{Patients}

Of the 639 patients with definite TIA in the standard care cohort, 392 met criteria for the "high risk standard care cohort". Of 204 patients admitted to the rapid evaluation unit during the period March 2002 to April 2003, 15 were excluded as they presented with ischemic stroke $(n=10)$ or were from out of province $(n=5)$, resulting in a total of 189 patients for final analysis.

\section{Baseline characteristics}

Presenting characteristics for patients in the standard care and rapid evaluation cohorts are displayed in Table 1 . There were greater proportions of males $(57.1 \%$ vs. $45 \%, \mathrm{p}<.01)$ and smokers $(50.8 \%$ vs. $25.7 \%, \mathrm{p}<.001)$ in the rapid evaluation cohort, but more patients with previously diagnosed congestive heart failure $(7.6 \%$ vs. $3.2 \%, \mathrm{p}<.01)$, and dementia $(5.1 \%$ vs. $1.6 \%, \mathrm{p}<.001)$ in the standard care cohort.

In terms of TIA symptom complex, there were also more patients with motor $(68.1 \%$ vs. $53.4 \%, \mathrm{p}<.01)$, speech $(49.7 \%$ vs. $34.4 \%, \mathrm{p}<.01)$, and visual symptoms $(10.5 \%$ vs. $2.1 \%, \mathrm{p}<.001)$ in the "high-risk" standard care cohort than in the rapid evaluation cohort. Significantly more patients in the rapid evaluation cohort 
Table 1: Baseline patient characteristics

\begin{tabular}{|c|c|c|c|c|}
\hline Variable & $\begin{array}{l}\text { Standard care } \\
\text { cohort with definite } \\
\text { TIA }(\%) \\
(\mathrm{n}=639)\end{array}$ & $\begin{array}{l}\text { High risk standard } \\
\text { care cohort }(\%) \\
(n=392)\end{array}$ & $\begin{array}{l}\text { Rapid eval } \\
\text { cohort }(\%) \\
(n=189) *\end{array}$ & $\begin{array}{l}\text { luation } \\
\text { ) } \\
\text { p value }\end{array}$ \\
\hline \multicolumn{5}{|l|}{ Patient demographics } \\
\hline Age (mean) & 70.1(19-98) & 71.0(19-98) & \multicolumn{2}{|c|}{$67.5(14-93)$} \\
\hline Female sex & $369(57.8)$ & $216(55.0)$ & $81(42.9)$ & $\mathrm{p}=.008$ \\
\hline \multicolumn{5}{|l|}{ Comorbid conditions } \\
\hline Diabetes & $113(17.7)$ & $79(20.2)$ & $28(14.8)$ & \\
\hline Hypertension & $376(57.4)$ & $236(60.2)$ & $123(65.1)$ & \\
\hline Hypercholesterolemia & $165(25.8)$ & $105(26.8)$ & $65(34.7)$ & \\
\hline Smoking & $211(33.0)$ & $140(25.7)$ & $97(50.8)$ & $\mathrm{p}<.001$ \\
\hline Coronary artery disease & $147(23.0)$ & $98(25.0)$ & $47(24.9)$ & \\
\hline Myocardial infarction & $96(15.0)$ & $65(16.6)$ & $40(21.2)$ & \\
\hline Previous TIA or stroke & $214(33.5)$ & $146(37.2)$ & $77(40.5)$ & \\
\hline Atrial fibrillation & $78(12.2)$ & $65(16.6)$ & $24(12.6)$ & \\
\hline Congestive heart failure & $36(5.6)$ & $30(7.6)$ & $6(3.2)$ & $\mathrm{p}=.04$ \\
\hline Valvular heart disease & $29(4.54)$ & $19(4.9)$ & $16(8.4)$ & \\
\hline Peripheral vascular disease & $38(6.0)$ & $27(6.9)$ & $8(4.2)$ & \\
\hline Chronic renal disease & $51(8.0)$ & $30(7.7)$ & $11(5.8)$ & \\
\hline Peptic ulcer disease & $27(4.2)$ & $17(4.3)$ & $3(1.6)$ & \\
\hline Connective tissue disease & $16(2.5)$ & $9(2.3)$ & 0 & \\
\hline Neoplasm & $60(9.4)$ & $44(11.2)$ & $21(11.1)$ & \\
\hline Dementia & $33(5.2)$ & $20(5.1)$ & $3(1.6)$ & $\mathrm{p}<.04$ \\
\hline \multicolumn{5}{|l|}{ TIA Symptom Complex } \\
\hline Motor symptoms & $267(42.8)$ & $267(68.1)$ & $101(53.4)$ & $\mathrm{p}=.001$ \\
\hline Aphasia/speech disturbance & $195(30.5)$ & $195(49.7)$ & $65(34.4)$ & $\mathrm{p}=.001$ \\
\hline Sensory symptoms & $266(41.6)$ & $121(30.9)$ & $57(30.2)$ & \\
\hline Monocular/hemi-field visual loss & $41(6.4)$ & $41(10.5)$ & $4(2.1)$ & $\mathrm{p}<.001$ \\
\hline Gait/balance disturbance & $69(10.8)$ & $32(8.2)$ & $15(8.0)$ & \\
\hline \multicolumn{5}{|l|}{$\begin{array}{l}\text { Baseline stroke prevention } \\
\text { medications }\end{array}$} \\
\hline ASA & $236(37.0)$ & $145(37.0)$ & $92(48.7)$ & $\mathrm{p}=.009$ \\
\hline Clopidigrel & $32(5.0)$ & $24(6.1)$ & $14(7.4)$ & \\
\hline Antihypertensive & $288(45.0)$ & $182(46.4)$ & $98(52.9)$ & \\
\hline Antihyperlipidemic & $79(12.4)$ & $47(12.0)$ & $40(21.1)$ & $\mathrm{p}=.006$ \\
\hline Diabetic medication & $71(11.1)$ & $50(12.8)$ & $17(9.0)$ & \\
\hline Warfarin & $53(8.3)$ & $41(10.5)$ & $10(5.3)$ & $\mathrm{p}=.04$ \\
\hline Estrogen derivative & $51(8.0)$ & $31(8.0)$ & $19(10.1)$ & \\
\hline
\end{tabular}

* Test of proportions between high-risk and TIA Reference Unit, Fisher's Exact Test, only significant values $(\mathrm{p}<.05)$ shown.

were on ASA $(48.7 \%$ vs. $37.0 \%$, p<.01), or an antihyperlipidemic drug $(21.1 \%$ vs. $12.0 \%)$ at baseline than in the high-risk standard care cohort, but more patients in the standard care cohort were on coumadin $(10.5 \%$ vs. $5.3 \%, \mathrm{p}<.001)$ compared to the rapid evaluation cohort.

\section{Patient management}

Table 2 displays the initial management of patients in the rapid evaluation cohort as compared to the standard care cohort. Compared to the standard care cohort, patients admitted to the rapid evaluation unit had significantly more magnetic resonance imaging and echocardiograms. The rapid evaluation cohort also received more specialized techniques for detecting carotid stenosis such as MR angiogram, and CT angiogram. With respect to new treatments, patients in the rapid evaluation cohort were more likely to receive a new therapy on discharge including more anti-hypertensive and cholesterol-lowering agents. Patients admitted to the rapid evaluation unit were also more likely to receive carotid endarterectomy or angioplasty.

\section{Etiology}

The percentage of strokes after TIA by etiology of TIA for patients in each cohort is illustrated in the Figure. The most common etiology was 'unknown', the final diagnosis for $41.6 \%$ of patients admitted to the rapid evaluation unit. There was significantly more patients diagnosed with carotid stenosis in the rapid evaluation cohort as compared to the standard care cohort $(42 / 189(22.2 \%)$ vs. 23/392(5.9\%) p<.001). 
Table 2: Investigations and resources used in initial management of TIA in patients admitted to the Rapid Evaluation Unit versus those in the 'high risk standard care cohort'.

\begin{tabular}{|c|c|c|c|}
\hline Variable & $\begin{array}{l}\text { High risk standard } \\
\text { care cohort }(\%) \\
\mathrm{N}=392\end{array}$ & $\begin{array}{l}\text { Rapid evaluation } \\
\text { cohort }(\%) \\
\mathrm{N}=189\end{array}$ & p-value* \\
\hline \multicolumn{4}{|l|}{$\begin{array}{l}\text { Investigations/Referrals } \\
\text { (within } 30 \text { days) }\end{array}$} \\
\hline ECG & $343(87.5)$ & $144(76.2)$ & .001 \\
\hline CT SCAN & $244(62.2)$ & $127(67.2)$ & \\
\hline MRI & $23(5.9)$ & $62(32.8)$ & $<.001$ \\
\hline Carotid doppler & $99(25.3)$ & $72(38.1)$ & .002 \\
\hline Holter Monitor & $60(15.3)$ & $34(17.8)$ & \\
\hline Echocardiogram & $89(22.7)$ & $75(39.7)$ & $<.001$ \\
\hline CT angiogram $\dagger$ & $0(0)$ & $64(33.9)$ & $<.001$ \\
\hline MR angiogram $\dagger$ & $16(4.1)$ & $68(36.0)$ & $<.001$ \\
\hline \multicolumn{4}{|l|}{ Institution of new therapy } \\
\hline Currently on ASA & $145(37.0)$ & $93(49.0)$ & .005 \\
\hline New prescription ASA & $131(33.4)$ & $68(36.0)$ & \\
\hline Increase in ASA & $49(12.5)$ & $1(0.01)$ & $<.001$ \\
\hline Clopidogrel & $38(9.7)$ & $21(11.1)$ & \\
\hline New Antihypertensive & $14(3.6)$ & $17(14.8)$ & .01 \\
\hline New Statin & $4(1.0)$ & $20(10.6)$ & $<.001$ \\
\hline Adjustment or initiation of coumadin & $24(6.1)$ & $18(9.5)$ & \\
\hline Carotid procedure(within 90 days) & $10(2.6)$ & $21(11.1)$ & $<.001$ \\
\hline Disposition & $84(21.4)$ & $189(100)$ & \\
\hline Admission & $122(31.1)$ & -- & \\
\hline Neurological consultation(ER) & $225(57.4)$ & -- & \\
\hline \multicolumn{4}{|l|}{ Referral to outpatient Stroke Prevention } \\
\hline Clinic & $154(39.4)$ & -- & \\
\hline $\begin{array}{l}\text { Patients actually Seen in Stroke Prevention } \\
\text { clinic } \\
\text { Patients with uncertain follow-up in next } 30 \\
\text { days }\end{array}$ & $156(39.8)$ & -- & \\
\hline
\end{tabular}

* p-value for comparison between high-risk standard care cohort and rapid evaluation cohort, Fisher's Exact test, only significant results $(\mathrm{p}<.05)$ shown; $\uparrow \mathrm{CT}$ angiogram and MR angiogram include carotid imaging early stroke as the dependent variable and treatment cohort, and baseline characteristics as independent variables. Using multivariable analysis, the major predictor associated with recurrence of TIA was the type of cohort; the rapid evaluation unit was associated with a decreased early risk of stroke (OR 0.43, p=0.029) compared to standard care. Male sex was the only other significant predictor for early stroke (OR 1.91, p=.04), after controlling for other variables of interest.

Costs of managing patients in the rapid evaluation unit vs. standard care

Year 1 median costs were significantly greater for the rapid evaluation cohort (CAN\$8360) as compared to the standard care cohort (CAN\$4820), ( $<<.001$, Kruskal-Wallis non-parametric test). The increase in costs for the rapid evaluation unit was mostly due to higher initial inpatient costs, but all median costs including physician billings, outpatient costs, and drug costs were higher for this cohort.

\section{DISCUSSION}

We report an unadjusted risk of early stroke for patients in the rapid evaluation cohort of $4.7 \%$ vs. $9.7 \%$ in

\section{Early risk of stroke after TIA}

Within 90 days of the TIA, stroke occurred in 38 of 392 patients $(9.7 \%)$ in the "high-risk" standard care cohort compared with 9 of 189 patients in the rapid evaluation cohort $(4.7 \%$; $\mathrm{p}=0.05)$. The Figure reports the occurrence of stroke according to etiology. Of note, there appeared to be a decreased early risk of stroke in those patients admitted to the rapid evaluation unit with the eventual diagnosis of carotid stenosis compared to those diagnosed with carotid stenosis in the standard care cohort $(5 \%$ vs. $26 \%, \mathrm{p}<0.019)$. This may have been due to the fact that more patients with carotid stenosis in the rapid evaluation unit received some form of carotid procedure (Table 3). For patients with known carotid stenosis, the proportion of patients receiving carotid endarterectomy or carotid stenting was similar between the cohorts; however, nearly $50 \%$ of patients in the standard care cohort received their procedure after suffering a recurrent stroke - in contrast, more procedures were done in the rapid evaluation cohort after TIA but before any recurrent stroke (Table 3).

To control for known differences in baseline characteristics, we also performed regression analysis using the occurrence of

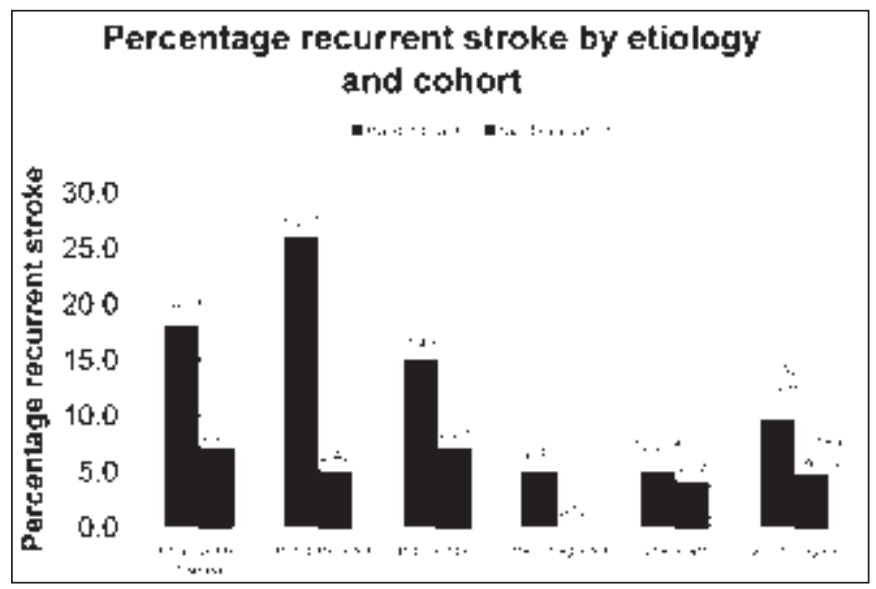

Figure: Percentage of recurrent stroke within 90 days by TIA etiology and cohort group. ${ }^{*}$ p-value $=0.019$, comparison for recurrent stroke in patients with known carotid stenosis between high-risk standard care cohort and rapid evaluation cohort, Fisher's Exact test; ** p-value $=0.05$, comparison for recurrent stroke for all patients between high-risk standard care cohort and rapid evaluation cohort, Fisher's Exact test. 
Table 3: Use of carotid intervention in patients in the rapid evaluation and standard care cohorts

\begin{tabular}{|c|c|c|}
\hline & $\begin{array}{l}\text { High-risk standard care cohort } \\
(\mathrm{n}=392)\end{array}$ & $\begin{array}{l}\text { Rapid evaluation cohort } \\
(\mathrm{n}=189)\end{array}$ \\
\hline $\begin{array}{l}\% \text { patients with carotid intervention within } 90 \text { days post } \\
\mathrm{TIA}\end{array}$ & $10 / 392(2.6 \%)$ & $21 / 189(11.1 \%)^{*}$ \\
\hline $\begin{array}{l}\text { Proportion of patients with carotid intervention within } 90 \\
\text { days (of those with known carotid disease) }\end{array}$ & $10 / 23(43 \%)$ & $21 / 42(50 \%)$ \\
\hline $\begin{array}{l}\text { Patients with carotid intervention occurring AFTER } \\
\text { recurrent stroke }\end{array}$ & $5 / 10(50 \%)$ & $0 / 21(0 \%) *$ \\
\hline Patients with early stroke following carotid intervention & $0 / 10(0 \%)$ & $1 / 21(5 \%)$ \\
\hline
\end{tabular}

the standard care cohort. The results of the logistic regression analysis confirmed that patients with high risk TIA in the rapid evaluation cohort had a lower incidence of early stroke even after controlling for baseline differences among the cohorts.

The purpose of admitting high risk patients is both to expedite investigations and to initiate immediate treatment. Previous attempts to manage these patients in an outpatient setting appear to have resulted in unacceptable delays and hence the occurrence of early strokes. By introducing a rapid evaluation unit, we identified proportionally more cases of carotid stenosis, a group known to be at high risk for early stroke, ${ }^{8}$ as compared to the standard care cohort. This may be due to clinical selection when admitting patients to the rapid evaluation unit or may be due to identifying more of these patients through intensive investigation. Within the rapid evaluation unit, many patients with high-risk TIA underwent neurovascular imaging such as CT angiography or MR angiography within the Emergency Department, which was used to expedite appropriate management.

Despite the greater number of patients identified with carotid stenosis in the rapid evaluation cohort, there was a significant decrease in number of early strokes recorded for this etiologic group as compared to the 'standard care' cohort. On average 10$12 \%$ of patients admitted to the Rapid Evaluation Unit with a motor or speech TIA underwent carotid revascularization and such patients had their procedure routinely completed within the first two weeks. When completed rapidly, carotid endarterectomy is known to be highly beneficial. ${ }^{9}$

Patients admitted but not requiring endarterectomy were more likely to be discharged on antihypertensive and lipid lowering agents, anti-platelet drugs and anticoagulants. It is not known whether such medications reduce the early risk of stroke but it is biologically plausible to expect that the combined approach of early aggressive risk factor management and antithrombotic therapy will reduce stroke risk. We speculate that the observed lower early risk of stroke was due to the combination of early aggressive carotid revascularization for eligible patients and risk factor management for all patients.
There are several strengths to this study. We carefully collected the data on exposure and outcome in a similar fashion for patients in both cohorts. With respect to exposure, it is clear which of the groups was 'exposed' or specifically attended the rapid evaluation unit and which did not, as the study involved a historical cohort. Furthermore, we minimized bias with respect to differential loss to follow-up. The follow-up period for recurrence of stroke was a short period of 90 days and we used several methods including chart review and use of provincial administrative data to collect data on the primary outcome. Data available from Alberta Health and Wellness confirmed that only a small percentage of patients $(<1 \%)$ were lost to follow-up due to migration out of Alberta during this time frame.

There are some limitations to this study which should be acknowledged. Admission to the rapid evaluation unit was lower than expected. It is probable that the referral process did not identify all eligible high risk patients in the year that the rapid evaluation unit was opened and this is supported by the noted differences in baseline characteristics between the two cohorts. However, all clinically relevant variables likely to 'confound' the analysis were included in the logistic regression model, and the main study results remained unchanged. Although we did not capture this group of patients who were not admitted to the rapid evaluation unit during this period (and may have been treated as outpatients), the early risk of stroke would have been expected to be lower for these un-referred patients given that high risk TIA patients would have been more likely to be referred for admission. This group of patients is a potential cohort for further study.

In addition, the use of a historical control group of patents does raise additional issues regarding treatment differences present over time which may introduce a bias in favour of the group with more current treatment. In fact, our study does confirm that rapid evaluation does appear to identify those high risk patients with carotid stenosis who need timely carotid intervention to prevent recurrent stroke, a management strategy that was not routinely applied to the standard care group. 
Finally, this was not a randomized clinical trial and there will always be unmeasured confounding variables that may affect the internal validity of the study. However, we attempted to identify comparable groups of patients by defining, a priori, a subgroup of historical patients who would have been eligible for the rapid evaluation unit had it been available at the time. Ideally, the effectiveness of a rapid evaluation unit for TIA requires verification in a randomized clinical trial. Although a randomized clinical trial would provide important evidence, designing such a trial would have a number of ethical barriers and would likely prove to be challenging.

Management of TIA within a rapid evaluation unit is associated with increased costs; the one-year median costs were approximately \$CAD3600 higher as compared to standard care. Based on our results, the approximate cost of preventing one stroke with the rapid evaluation unit is grossly estimated at \$CAD72000 after one year. It is clear that the total lifetime cost of caring for ischemic stroke survivors is a significant economic burden with estimates ranging between $\$ U S 19,000$ to \$US230,000 (1990 values) depending upon costing methods used. ${ }^{10}$ Thus, conceivably, the initial higher costs of caring for TIA patients through a rapid evaluation unit may be offset by the averted long-term cost of caring for patients with disabling strokes.

In summary, the results of this study show an association between intervention in a rapid evaluation unit and a lower early risk of stroke. We highlight the need for further study of rapid evaluation methods for patients with high risk TIA which also incorporate formal economic evaluation.

\section{SOURCES OF FUNDING}

This study was funded by a grant from the Adult Research Committee of the Calgary Health Region. Fellowship support for CMW was provided through the Canadian Institutes for Health Research and the Alberta Heritage Foundation for Medical Research.

\section{REFERENCES}

1. Johnston SC, Fayad P, Gorelick P, Hanley D, Shwayder P, van Husen D, et al. Prevalence and knowledge of transient ischemic attacks among US adults. Neurology. 2003;60:1429-34.

2. Johnston SC, Gress DR, Browner WS, Sidney S. Short-term prognosis after emergency department diagnosis of TIA. JAMA. 2000;284:2901-06.

3. Gladstone DJ, Kapral MK, Fang J, Laupacis A, Tu JV. Management and outcomes of transient ischemic attacks in Ontario. CMAJ. 2004;170(7):1099-104.

4. Hill MD, Yiannokoulias N, Jeerakathil T, Tu JV, Lawrence LW, SvensonLW, et al. The high risk of stroke immediately after transient ischemic attack; a population study. Neurology. 2004; 62: $2015-20$

5. Wu CM, McLaughlin K, Lorenzetti DL, Hill MD, Manns BJ, Ghali WA. Early risk of stroke after transient ischemic attack: a systematic review and meta-analysis. Arch Intern Med. 2007; 167:2417-22.

6. Rothwell PM, Giles MF, Chandratheva A, Marquadt L, Geraghty O, Redgrave NE, et al. on behalf of the Early Use of Existing Preventative Strategies for Stroke (EXPRESS) study. Effect of urgent treatment of transient ischemic attack and minor stroke on early recurrent stroke (EXPRESS study): a prospective population-based sequential comparison. Lancet. 2007;370: 1432-42.

7. Lavallee PC, Meseguer E, Abboud H, Cabrejo L, Olivot J, Simon $\mathrm{O}$, et al. A transient ischemic attack clinic with round -the-clock access (SOS-TIA): feasibility and effects. Lancet Neurol. 2007;6:953-60.

8. Eliasziw M, Kennedy J, Hill MD, Buchan AM, Barnett HJM, for the North American Symptomatic Carotid Endarterectomy Trial (NASCET) group. Early risk of stroke after a transient ischemic attack in patients with internal carotid artery disease. CMAJ. 2004;170(7):1105-9.

9. Rothwell PM, Eliasziw M, Gutniknov SA, Warlow CP, Barnett HJM, for the Carotid Endarterectomy Trialists Collaboration. Endarterectomy for symptomatic carotid stenosis in relation to clinical subgroups and timing of surgery. Lancet. 2004;363: 915-24.

10. Payne KA, Huybrechts KF, Caro JJ, Craig Green TJ, Klittich WS. Long-term cost-of illness in stroke. An international review. Pharmacoeconomics. 2002;20(12):813-25. 